\title{
Crack Length Based Healing Characterisation of Bitumen at Different Levels of Cracking Damage*
}

\section{Linglin Li, Ph.D.}

Associate Professor ${ }^{1} \&$ Marie Skłodowska-Curie Research Fellow ${ }^{2}$

${ }^{1}$ Automotive and Transportation Engineering College

Hefei University of Technology, Hefei, Anhui, China, 230009

${ }^{2}$ Aston Institute of Materials Research

School of Engineering and Applied Science

Aston University, MB226, Aston Triangle, Birmingham, B4 7ET, U.K.

Email:1.li28@aston.ac.uk

Yangming Gao, Ph.D. Candidate

Aston Institute of Materials Research

School of Engineering and Applied Science

Aston University, MB226, Aston Triangle, Birmingham, B4 7ET, U.K.

Email: gaoy14@aston.ac.uk

Yuqing Zhang, Ph.D.

Senior Lecturer in Highway Engineering, Engineering Systems \& Management Group (ESM)

Deputy Director, Aston Institute of Materials Research

Aston University, MB226E, Aston Triangle, Birmingham, B4 7ET, U.K.

Email: y.zhang10@aston.ac.uk

(Corresponding Author)

Word Count: 7746

Submitted [18/10/2019]

* This is an Accepted Manuscript of an article published by Journal of Cleaner Production. Access to the full text of the paper is available at https://doi.org/10.1016/j.jclepro.2020.120709 


\title{
Crack Length Based Healing Characterisation of Bitumen at Different Levels of Cracking Damage
}

\author{
Linglin $\mathrm{Li}^{\mathrm{a}}{ }^{\mathrm{b}} \mathrm{b}$ Yangming Gao ${ }^{\mathrm{b}}$ and Yuqing Zhang ${ }^{\mathrm{b}, \uparrow}$ \\ ${ }^{a}$ School of Automotive \& Transportation Engineering, Hefei University of Technology, Hefei, China \\ ${ }^{\mathrm{b}}$ Aston Institute of Materials Research, Aston University, Birmingham, United Kingdom
}

\begin{abstract}
:
This study aims to characterise the healing properties of asphalt binders at different damage levels. The healing and the damage levels were quantified by crack length $(C L)$ in binder samples generated by rotational shear fatigue loads in strain-controlled dynamic shear rheometer (DSR) tests. For comparison, the healing was also characterised by the commonly used material parameters including pseudo shear stiffness $(S)$ and dissipated pseudo strain energy (DPSE). A normalized healing index was formulated using the above three parameters, respectively. A healing test of polymer-modified bitumen was designed based on DSR including a straincontrolled time sweep test plus a rest duration and followed by another strain-controlled time sweep test. The healing tests were performed at different rest start times $(5 \mathrm{~min}, 10 \mathrm{~min}$ and $20 \mathrm{~min})$, rest durations ( $5 \mathrm{~s}, 10 \mathrm{~s}, 0.5 \mathrm{~min}, 1 \mathrm{~min}, 2 \mathrm{~min}, 5 \mathrm{~min}, 10 \mathrm{~min}, 20 \mathrm{~min}$, and $40 \mathrm{~min}$ ), and amplitudes of the shear strain $(5 \%, 7 \%$, and $10 \%)$ at $20^{\circ} \mathrm{C}$ and $10 \mathrm{~Hz}$. Results show that the $C L$ based healing index is a fundamental and accurate parameter to evaluate the healing rate and healing potential of the bitumen. DPSE-based healing index is applicable only when the energy is mainly dissipated to generate cracks. Healing is underestimated when characterised using $S$ or DPSE-based healing indices. Healing index increases due to the advance of rest duration or the decrease of the damage level, and the healing rate can be essentially modelled by RambergOsgood model. Higher damage levels (introduced by higher load levels or longer loading time) can effectively decrease the binders' healing potentials when the binders are at the relatively low damaged levels. The healing potential becomes low when the material is at severe damage state, thus will remain at that low levels even though the damage level increases.
\end{abstract}

Keywords: Bitumen, DSR, Healing Index, Healing Rate, Crack Length, Damage Level

\footnotetext{
${ }^{\dagger}$ Corresponding author.

E-mail address: 1.li28@aston.ac.uk (Linglin Li), gaoy14@aston.ac.uk (Yangming Gao), y.zhang10@aston.ac.uk (Yuqing Zhang)
} 


\section{Introduction}

Bitumen, a by-product of crude oil distillation, is heavily used for constructing the surface layers of highways, bridges and runways of airport. It is susceptible to damage in the form of cracks, leading to mechanical degradation. The bitumen has an advantageous ability to partially or fully heal the cracks by itself (Qiu et al., 2011). However, this healing ability of the bitumen is typically not accounted for performance prediction of the material, thus the mechanical degradation of the bitumen is normally overestimated (Ayar et al., 2016). The overestimated material degradation has resulted in a conservative pavement structural design and the waste of construction materials such as bitumen and asphalt. Thus, addressing the healing effect of the bitumen in material selection and structural design has been a new focus and a non-negligible step in sustainable pavement design.

The healing phenomenon was initially observed in bitumen and asphalt pavement in 1960s (Bazin and Saunier, 1967). Until recent, how to enhance the healing rate (i.e., speed) and healing potential (i.e., magnitude) has been a research focus in both laboratory and field scales (Koohi et al., 2013; Liu et al., 2017; Luo et al., 2019). Particularly, when the healing potential is fully triggered and the healing rate is effectively accelerated, bitumen can essentially heal all the micro cracks in the asphalt pavement. This will dramatically reduce the maintenance costs (Tabakovic and Schlangen, 2016), extend the service life (Patti et al., 2018) and eventually preserve the natural resources (i.e., bitumen and minerals) and fossil fuels and minimise the emission of greenhouse gas due to less re-construction and maintenance work of the road infrastructure.

The healing of bituminous materials is normally characterised by a healing index, which is a percent ratio of the recovery of a material parameter after a rest period (i.e., a healing duration with no external load applied) to the material parameter before the rest period. The selection of the material parameter for healing characterisation is very empirical based. Kim et al. (1990) and Little et al. (1999) evaluated the healing ability of an asphalt mixture in a strain-controlled test and quantified the healing using dissipated pseudo strain energy (DPSE). Their healing index was defined as the ratio of the recovered DPSE after the rest period to the one prior to the rest period. Si et al. (2002) used pseudo stiffness to quantify the damage of asphalt mixture, which decreased consistently with an increased number of loading cycles and recovered notably after a rest duration was introduced. Therefore, they defined healing index as the ratio of recovered pseudo stiffness following the rest period to the pseudo stiffness prior to the rest period. Tan et. al (2012) defined healing index based on dynamic shear modulus or specific load cycles. García (2012) employed the ultimate (the peak) force of an asphalt mastic beam in a three-point flexural bending test to define the healing index. Li et. al (2018) employed dynamic shear modulus, fatigue life (defined as the load cycle when module dropped by $50 \%$ ) and dissipated energy to quantify healing index, respectively. Ganjei and Aflaki (2019) decomposed a total fracture energy of an asphalt mixture under cyclic load into dissipated creep strain energy (DCSE) and elastic energy. They defined healing index as the ratio of the recovered DCSE during the rest period to the total decrease of the DCSE in the whole fatigue period. It is evident that no common conclusion was drawn on which material parameter should be used in defining the healing index. As Little (1999) interpreted, healing index is just an empirical indication of the rate at which healing proceeds. Because of its empirical nature, the healing index for the same material can be significantly different when different material parameters are used. It is proposed in this study that the healing in bituminous material should be directly defined based on crack length as the healing is a process of crack reduction which results in the recovery of the other material properties. Thus, there is an urgent research need to characterise the healing in bituminous material directly based on crack length such that the accurate healing can be 
quantified.

As an inherent property of bitumen, healing can be affected by internal material factors and external loading and environmental factors. The internal factors mainly include surface energy and material chemical composition. Cheng et al. (2002) found that cohesive and adhesive healing within the bitumen-aggregate systems were directly associated with the surface energies of bitumen and aggregates. Sun et al. (2017) employed TLC, GPC, FTIR, and NMR to determine four bitumen binders' chemical compositions which are found to significantly affect the healing capability. The external factors affecting bitumen's healing performance include rest period, compressive stress, temperature, moisture and ageing. Lee et al. (2011) investigated the healing properties by rest periods on the slab of asphalt mixture by Model Mobile Loading Simulator and Nondestructive Evaluation techniques. Qiu et al. (2011) observed that the compressive stress applied on the crack surfaces can strongly enhance the healing speed. Liu et al. (2016) concluded that thermal activated healing of micro cracks was clearly practical to extend the fatigue life of bitumen and different kinds of bitumen needed to be heated to specific softening point related temperatures to substantially enhance the healing ability and rate. Mannan et al. (2017) found that moisture diffused into the bitumen can reduce the value of short-term healing by lessening the cohesive energy used to crack the bitumen, and decrease the value of long-term healing by raising the required activation energy for bituminous molecular diffusion. Norambuena-Contreras et al. (2018) found that ageing levels can evidently degrade the healing effect of asphalt mixture. It is widely believed that only micro-crack can be effectively healed, and the healing potential and rate of asphalt can be dramatically reduced as the crack evolved from micro-crack to macrocrack (Kringos et al., 2009). However, these were mainly laboratory observations or modelling predictions, and their lack of solid data to quantify how the damage level will affect the healing process. Therefore, how different levels of crack damage affects the healing potential and healing rate of bitumen is another critical issue needing addressed.

This study aims to characterise the healing properties of bitumen based on crack length $(C L)$ and evaluate the impact of the cracking damage levels on the healing performance of the bitumen. In comparison, the healing of the bitumen was also defined by pseudo stiffness $(S)$ and dissipated pseudo strain energy (DPSE). A healing test on a polymer-modified bitumen $\mathrm{X} 70$ was conducted using dynamic shear rheometer (DSR). The $C L$-based healing index was justified against the healing indices defined by the $S$ and DPSE under different rest durations and cracking damage levels. Finally, Ramberg-Osgood model was utilised to model the healing rate, where short-term and long-term healing rates and the healing potential were obtained at different damage levels.

\section{Model Bitumen's Healing Using Different Material Parameters}

\subsection{Material Parameters to Characterise Healing of Bitumen}

Healing of bitumen has been intensively investigated for decades and many phenomenological methods are used to characterise the healing by the recovery of material parameters. Dynamic shear modulus is commonly utilised as one of key viscoelastic parameters to characterise the rheological properties of the bitumen. Moreover, as the damage (i.e. crack) of the bitumen sample initiates and propagates, the reduction of the dynamic shear modulus starts and continues. Therefore, dynamic shear modulus is naturally selected as a candidate material parameter to characterise healing properties by many existing studies (Canestrari et al., 2015; Tan et al., 2012). However, phase angle is another independent viscoelastic parameter that cannot be neglected when quantifying the recovery of material properties as healing happens in the viscoelastic bituminous materials. This implies that dynamic shear modulus, by itself, may not be able to accurately and comprehensively 
quantify the healing in bituminous material. Fundamentally, this is due to a fact that the viscoelastic relaxation and healing of a viscoelastic material such as bituminous material can both apparently lead to the recovery of the dynamic shear modulus during the healing rest period. It is necessary to differentiate the modulus recovery due to the relaxation and that due to healing of cracks, in order to accurately characterise healing in a viscoelastic material. This differentiation can be achieved by pseudo shear stiffness, which justifies the use of pseudo shear stiffness for healing characterisation in the literature. Therefore, pseudo shear stiffness, rather than the dynamic shear modulus, will be utilised as one of the material parameters to formulate healing index in this paper.

Apart from dynamic modulus, many other material parameters, such as pseudo (shear) stiffness (Si, Z. et al., 2002), internal state variable (Palvadi et al., 2012), dissipated strain energy (Carpenter and Shen, 2006), dissipated pseudo strain energy and damage density (mainly used to assess the damage and healing property of asphalt mixture) (Lytton et al., 2001), were employed to characterise the healing behaviour of the bituminous materials. In this study, pseudo shear stiffness and dissipated pseudo strain energy will be employed to define the healing index and be used for comparison with the healing index defined by cracking length, since both were able to differentiate the healing from the viscoelastic relaxation and both were widely used in the existing studies in the literature.

Pseudo shear stiffness $(S)$, a kind of secant modulus, is defined as follows (Si, Z. et al., 2002):

$$
S=\frac{\tau_{m}}{\gamma_{m}^{R}}
$$

where, $\gamma_{m}^{R}$ is the peak pseudo shear strain in each load cycle; and $\tau_{m}$ is the shear stress corresponding to the $\gamma_{m}^{R}$ in the stress against pseudo shear strain diagram.

In a strain-controlled DSR test, shear strain $\chi(t)=\gamma_{d} \sin (\omega t)$ with $\gamma_{d}$ being the magnitude of the shear strain (on the sample edge of the DSR bitumen specimen) where a constant value (e.g. $5 \%$ ) is used in this study, and $\omega$ equalling to a selected loading frequency in $\mathrm{rad} / \mathrm{sec}$. According to the definition of pseudo shear strain, it can be written as:

$$
\begin{aligned}
\gamma^{R}(t) & =\frac{1}{G_{R}} \int_{0^{-}}^{t} G(t-s) \frac{\partial \gamma(s)}{\partial s} d s \\
& =\frac{\gamma_{d}}{G_{R}}\left|G_{0}^{*}\right| \sin \left(\omega t+\delta_{0}\right)
\end{aligned}
$$

where, $t$ is present time; $\gamma^{R}(t)$ is pseudo shear strain; $G_{R}$ is reference shear modulus and selected as a unit in this paper; $s$ is time history at which strain is measured; $G(t-s)$ is relaxation shear modulus in an undamaged state; $\chi \chi(s)$ is measured shear strain at previous time $s ; \omega$ is frequency; $\left|G_{0}^{*}\right|$ and $\delta_{0}$ are dynamic shear modulus and phase angle at the undamaged state, respectively; $\gamma_{d}$ is amplitude of shear strain.

Hence, the shear fatigue loading time corresponding to the peak pseudo shear strain $\gamma_{m}^{R}$ is calculated when $\omega t=\pi / 2+2 N \pi-\delta_{0}$ ( $N$ is the number of shear loading cycles) in the $N^{\text {th }}$ load cycle. The shear stress becomes $\tau(t)=\tau_{N} \sin \left(\omega t+\delta_{N}\right)$ with $\tau_{N}$ being the amplitude of shear stress where $\tau_{N}=\left|G_{N}^{*}\right| \gamma_{d}$, and $\delta_{N}$ equalling to the phase angle at the $N^{\text {th }}$ shear fatigue loading cycle. Using Eq. (1) and applying $\omega t=\pi / 2+2 N \pi-\delta_{0}$, pseudo shear stiffness is calculated as: 


$$
S=\left|G_{N}^{*}\right| G_{R} \cos \left(\delta_{N}-\delta_{0}\right) /\left|G_{0}^{*}\right|
$$

where, $\left|G_{N}^{*}\right|$ and $\delta_{N}$ are dynamic shear modulus and phase angle at the $N^{\text {th }}$ load cycle at the damaged state, respectively.

When plotting the measured stress versus the pseudo strain for bitumen, a hysteresis loop exists, the area of which is the dissipated pseudo strain energy (DPSE). The DPSE is mathematically interpreted as the difference between the total dissipated strain energy and dissipated strain energy due to the linear viscoelastic relaxation. In the $N^{\text {th }}$ shear load cycle, the total DPSE over the whole sample volume is calculated as:

$$
\begin{aligned}
\operatorname{DPSE}_{N} & =\iiint_{V_{0}} \int_{(N-1) T}^{N T} \tau(t) d \gamma^{R}(t) d V \\
& =\frac{\pi^{2}}{2} r_{0}^{2} \gamma^{2} h \frac{\left|G_{0}^{*}\right|}{G_{R}}\left|G_{N}^{*}\right| \sin \left(\delta_{N}-\delta_{0}\right)
\end{aligned}
$$

where, $T$ is the time period of the shear load cycle; $\tau(t)$ is shear stress; $r_{0}$ and $h$ are the radius and height of the DSR sample, respectively; $V_{0}$ is the volume of the DSR sample.

Healing index characterised by pseudo shear stiffness or dissipated pseudo strain energy is an empirical measurement of the material property (stiffness or strain energy) recovery of the bitumen during the healing rest period. It appears that the healing index defined by the $S$ or DPSE will only provide an indirect estimation of the cracking damage recovery in the bitumen. In a DSR fatigue test, the direct proof of the cracking damage is a circumferential edge crack, as illustrated in Figure 1. It initiates from the DSR sample circumferential edge and propagates toward the centre of the sample under the cyclic torsional loading, which were directly proved experimentally by the previous studies (Hintz and Bahia, 2013; Zhang and Gao, 2019). Thus, it is reasonable to define the healing index using the cracking length, since the healing is a process of crack reduction. More importantly, defining the healing using cracking length will lead to a significant convenience when coupling healing into a damage constitutive model. Based on damage mechanics, a damage density $\xi$ is defined as a lost material area (cracking area) divided by the total cross-sectional area. The damage density has been successfully utilised to capture the overall damage properties and relate the apparent material response to the effective responses and the fundamental material properties. When the healing index is defined using crack length (or cracking area), an analogous method can be directly used to incorporate healing index into the damage density to model the healing effect on damage responses in the constitutive modelling. Therefore, this study will propose a method to determine the healing index by crack length.

Figure 1 shows the trimmed configuration of an undamaged bitumen sample and also shows a schematic side view of a cylindrical damaged sample in the DSR tests, where $r \mathrm{E}$, and $c$ are the effective radius (the radius of the intact area of the damaged bitumen sample) and crack length at the $N^{\text {th }}$ load cycle. 

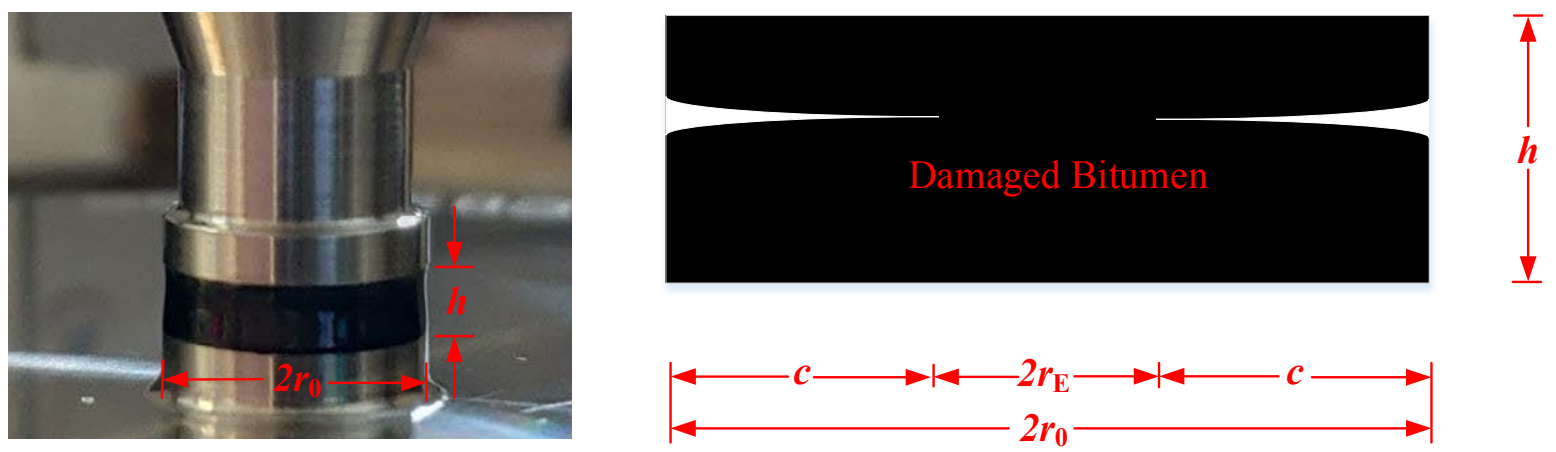

Fig. 1. Trimmed configuration of an undamaged cylindrical bitumen sample and schematic side view of the damaged cylindrical bitumen sample in DSR tests

Based on damage mechanics, two equilibrium principles (i.e., torque and dissipated strain energy) are employed to determine the crack length under a rotational shear fatigue load (in a strain-controlled time sweep procedure) (Zhang and Gao, 2019):

$$
c=\left\{1-\left[\frac{\left|G_{N}^{*}\right| / \sin \left(\delta_{N}\right)}{\left|G_{0}^{*}\right| / \sin \left(\delta_{0}\right)}\right]^{1 / 4}\right\} r_{0}
$$

where, $c$ is the crack length of the bitumen at the $N^{\text {th }}$ load cycle.

Equation (5) gives a mechanics-based approach to directly determine the crack length of a bitumen in a DSR test using the material properties such as shear modulus and phase angle. It is stressed that $\left|G_{0}^{*}\right|$ and $\delta_{0}$ (dynamic shear modulus and phase angle at the undamaged state, respectively) can be obtained at relatively low strain level (e.g. less than 1\%) and room temperature; $\left|G_{N}^{*}\right|$ and $\delta_{N}$ (dynamic shear modulus and phase angle at the damaged state, respectively) can be measured at high strain level (e.g. more than 5\%) and room temperature. Equation (5) was experimentally validated (Zhang and Gao, 2019). The crack lengths after the time sweep tests were measured using digital visualisation of cracking surfaces for one neat bitumen and one polymer-modified bitumen at two temperatures $\left(15,20^{\circ} \mathrm{C}\right)$, two frequencies $(10,20 \mathrm{~Hz})$ and two strain levels $(5 \%, 7 \%)$. Results show that the DSR-based crack growth model (i.e., Equation (5)) can accurately predict the crack length in the viscoelastic bitumen under the rotational shear fatigue load and at different loading and material conditions (Zhang and Gao, 2019).

According to Equations (3), (4), and (5), the S, DPSE, and CL were calculated for a straincontrolled time sweep healing test which includes a strain-controlled time sweep fatigue test for selected load cycles (i.e., 3000, 6000 and 12000), a healing rest period (e.g., 20min) and another time sweep fatigue test after the rest. Figure 2 presented the typical results of the $S$, $D P S E$, and $C L$ changing with the loading time. 


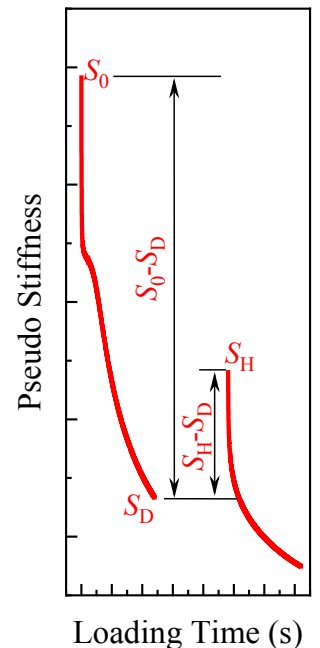

(a)

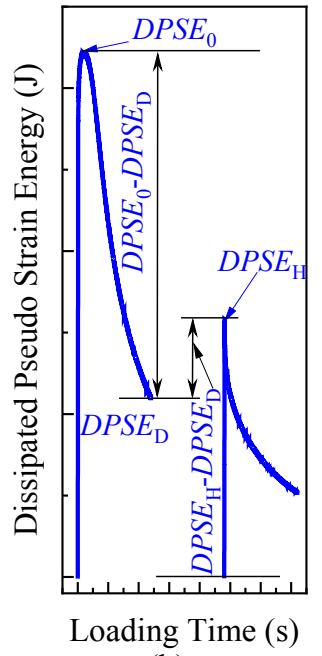

(b)

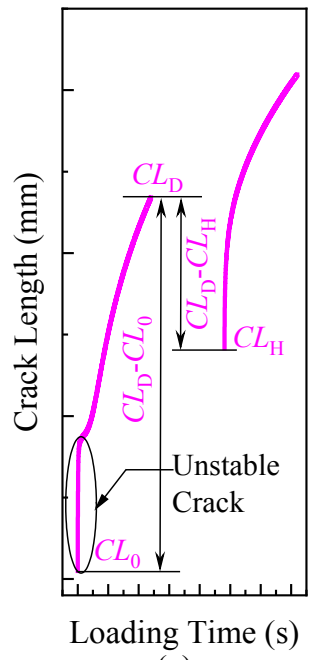

(c)

Fig. 2. Pseudo stiffness, dissipated pseudo strain energy and crack length in a straincontrolled time sweep healing test

Figure 2 (a) shows the pseudo shear stiffness $(S)$ determined by Equation (3) in the time sweep healing test. It is shown that as the time sweep test continues in the first part, the $S$ consistently decreases until the rest period is introduced. The loss of $S$ in the first part of the time sweep test is $S_{0}-S_{D}$ where $S_{0}$ and $S_{D}$ stands for the initial and the final values of $S$ before the rest period, respectively. As the rest period proceeds, the recovery of $S$ is expected and determined as $S_{H-} S_{D}$, where $S_{H}$ denotes the pseudo shear stiffness after healing which is also the initial pseudo stiffness at the beginning of the second part of the time sweep test.

Figure 2 (b) shows the dissipated pseudo strain energy (DPSE) determined by Equation (4) in the time sweep healing test. It is shown that the DPSE quickly grows from zero to a global maximum (i.e., $D P S E_{0}$ ) and then progressively drops to $D P S E_{D}$ until the fatigue load ceases before the rest period. According to the physical meaning of the DPSE and the findings from the previous study (Zhang and Gao, 2019), it is believed that the quick growth of DPSE from zero to $D P S E_{0}$ is consumed mainly for crack initiation to develop the initial disconnected micro-cracks at the edge of bitumen sample, which is unstable and dependent of sample edge shapes. After the crack initiation, the DPSE is primarily used for different physics including crack propagation, nonlinear viscoelasticity and heat loss due to shear rotational friction on cracking surfaces. When the rest duration is introduced, the DPSE steadily recovers from $D P S E_{D}$ (i.e., the $D S P E$ prior to the rest period) to $D P S E_{H}$ (i.e., the local maximum value of the DPSE after the rest period and in the second part of the time sweep test). Note that the reinitiation of cracks in the second part of the time sweep test (after the rest period) is very quick due to the stress concentration caused by the existing (unhealed) cracks in the samples. Therefore, one can find a sharp increase of the DPSE from a small value to DPSE $E_{H}$ after the healing rest period in Figure 2 (b). Hence, before the rest duration, the consumed DPSE is represented by $D P S E_{0}-D P S E_{D}$, and after the rest duration, the regained DPSE equals to $D P S E_{H}-D P S E_{D}$.

Figure 2 (c) shows the crack length (CL) determined by Equation (5) in the time sweep fatigue test. It clearly demonstrates the unstable crack in the initial stage of the crack growth, which predominantly depends on the microstructure and flow properties of the bitumen sample and implies the deterioration of the bitumen material. At the end of the first part of the time sweep test, the fatigue load yields a crack length of $C L_{D}$. After the healing rest period, the $C L$ reduced to $C L_{H}$. Accordingly, the length of crack healed by the rest duration is $C L_{D^{-}}$ $C L_{H}$. 


\subsection{Healing Index Models}

The pseudo stiffness, dissipated pseudo strain energy and crack length are employed herein to formulate a healing index which is used for quantifying the extension of healing performance of the bitumen material. The healing index $(\% H I)$ is defined as

$$
\% H I=\left|\frac{M_{H}-M_{D}}{M_{0}-M_{D}}\right| \times 100 \%
$$

where, $\% H I$ is the healing index; $M_{H}$ is the $S_{\mathrm{H}}, D P S E_{\mathrm{H}}$ or $C L_{\mathrm{H}}$ after the healing rest period as shown in Figure $2 ; M_{D}$ is the $S_{D}, D P S E_{D}$ or $C L_{D}$ at the last load cycle prior to the rest period; and $M_{0}$ is the $S_{0}, D P S E_{0}$ or $C L_{0}$ at the initial material parameters as shown in Figure 2.

By substituting Equation (3) into (6), one will have the $S$-based healing index:

$$
\% H I_{S}=\frac{\left|G_{H}^{*}\right| \cos \left(\delta_{H}-\delta_{0}\right)-\left|G_{D}^{*}\right| \cos \left(\delta_{D}-\delta_{0}\right)}{\left|G_{0}^{*}\right|-\left|G_{D}^{*}\right| \cos \left(\delta_{D}-\delta_{0}\right)} \times 100 \%
$$

where, $\left|G_{H}^{*}\right|$ and $\delta_{H}$ are the dynamic shear modulus and phase angle right after the rest period; $\left|G_{D}^{*}\right|$ and $\delta_{D}$ are the dynamic shear modulus and phase angle in the last load cycle prior to the rest period, respectively.

Substituting Equation (4) into (6), one will have the DPSE-based healing index:

$$
\% H I=\frac{D P S E_{H}-D P S E_{D}}{D P S E_{0}-D P S E_{D}} \times 100 \%
$$

The authors investigate both the short-term and long-term behaviours of bitumen healing at $20^{\circ} \mathrm{C}$. It is very difficult, if not impossible, to measure the healed crack length after short-term healing time (e.g., $5 \mathrm{~s}, 10 \mathrm{~s}$ ) at $20^{\circ} \mathrm{C}$. Hence, the new method based on Equation (5) is recommended to substitute the direct measurement method, because only dynamic modulus and phase angle after healing are needed to calculate healed crack length, which essentially originates from wetting diffusion, and randomization of bitumen chains (Wool and O'connor, 1981). Substituting Equation (5) into Equation (6), one will have the $C L$-based healing index:

$$
\% H I_{C}=\frac{\left[\left|G_{H}^{*}\right| / \sin \left(\delta_{H}\right)\right]^{1 / 4}-\left[\left|G_{D}^{*}\right| / \sin \left(\delta_{D}\right)\right]^{1 / 4}}{\left[\left|G_{0}^{*}\right| / \sin \left(\delta_{0}\right)\right]^{1 / 4}-\left[\left|G_{D}^{*}\right| / \sin \left(\delta_{D}\right)\right]^{1 / 4}} \times 100 \%
$$

Equations (7) and (9) show that when the dynamic moduli and phase angles of bitumen are measured from the time sweep healing test, the $S$-based healing index and the $C L$-based healing index can be directly obtained. When the DPSE is calculated by Equation (4), the $D P S E$-based healing index can then be determined using Equation (8).

\subsection{Healing Rate Model}

From the perspective of thermodynamics, healing of asphalt binder depends on its surface energy, in which the non-polar component $\left(\Gamma^{\mathrm{LW}}\right)$ sources from Lifshitz-Van der Waals force and the polar component $\left(\Gamma^{\mathrm{AB}}\right)$ results from Lewis acid-base force. There exist two healing mechanisms including a short-term and a long-term healing in its whole process. Lytton (2000) found that the short-term healing rate $\left(\dot{h}_{1}\right)$ depends primarily on $1 / \Gamma^{\mathrm{LW}}$, and the long- 
term healing rate $\left(\dot{h}_{2}\right)$ depends mainly on $\Gamma^{\mathrm{AB}}$. Based on the above observations, Lytton proposed two useful equations to calculate $\dot{h}_{1}$ and $\dot{h}_{2}$. Many researchers (Cheng et al., 2002; Luo and Lytton, 2015; Si, Z.S. et al., 2002) noted that the two healing rates occur simultaneously, and the real healing mechanism is the result of coactions of the above two healing rates. The actual healing rate $d(H I) / d t$ can be expressed by Ramberg-Osgood model (Ramberg and Osgood, 1943):

$$
\frac{d(H I)}{d t}=\dot{h}_{2}+\frac{\dot{h}_{1}-\dot{h}_{2}}{1+\frac{\dot{h}_{1}-\dot{h}_{2}}{h_{\beta}}(\Delta t)_{h}}
$$

where, $(\Delta t)_{h}$ is the rest period between load applications; and $h_{\beta}$ is a factor that varies between 0 and 1 and represents the healing potential, which is the maximum percent of bituminous healing that can be achieved. The value of $h_{\beta}$ is also empirically found to be related with $\Gamma^{\mathrm{AB} / \Gamma^{\mathrm{LW}}}$.

\section{Experimental Protocol and Plan}

\subsection{Materials}

A polymer-modified bitumen (X70), classed as 40/100-45 according to BSEN 14023:2010, was used in this study. The bitumen X70 comprises a range of elastomerically modified bitumen and can provide high performance over a range of different applications and conditions. Table 1 gives the detailed specification of the bitumen X70.

Table 1 Specification of polymer-modified bitumen X70

\begin{tabular}{|l|l|l|l|l|}
\hline Characteristic & Method & Unit & $\begin{array}{l}\text { Class according to } \\
\text { EN 14023:2010 }\end{array}$ & Value \\
\hline Penetration @2 25 $5^{\circ} \mathrm{C}$ & EN 1426 & dmm & 4 & $45-80$ \\
\hline Softening Point & EN 1427 & ${ }^{\circ} \mathrm{C}$ & 9 & $\geq 45$ \\
\hline Force Ductility @ $5^{\circ} \mathrm{C}$ & EN13589/13703 & $\mathrm{J} / \mathrm{cm}^{2}$ & 2 & $>3$ \\
\hline Change of Mass & EN $12607-1$ & $\%$ & 2 & $<0.3$ \\
\hline Retained Penetration & EN 1426 & $\%$ & 7 & $>60$ \\
\hline Increase in Softening Point & EN 1247 & ${ }^{\circ} \mathrm{C}$ & 2 & $<8$ \\
\hline Flash Point (Cleveland) & EN ISO 2592 & ${ }^{\circ} \mathrm{C}$ & 2 & $>250$ \\
\hline Fraas Breaking Point & EN 12593 & ${ }^{\circ} \mathrm{C}$ & 6 & $\leq-12$ \\
\hline Elastic Recovery @ $25^{\circ} \mathrm{C}$ & EN 13398 & $\%$ & 4 & $\geq 60$ \\
\hline Elastic Recovery @ $10^{\circ} \mathrm{C}$ & EN 13398 & $\%$ & 3 & $\geq 50$ \\
\hline $\begin{array}{l}\text { Storage Stability }(\text { Change of } \\
\text { Softening Point) }\end{array}$ & EN 13399 & $\%$ & 2 & $<5$ \\
\hline $\begin{array}{l}\text { Storage Stability (Change of } \\
\text { Penetration) }\end{array}$ & EN 13399 & $\mathrm{dmm}$ & 2 & $<9$ \\
\hline
\end{tabular}

\subsection{Test Method and Plan}

As described by Section 2, the main objective of the test is to obtain dynamic moduli and phase angles of the bitumen samples at different stages of fatigue damage and healing conditions. Before the start of healing test, the bitumen samples stored in the jar were heated in the laboratory oven at $165^{\circ} \mathrm{C}$ for $30 \mathrm{~min}$. Then hot bitumen was distributed into small containers and sampled for healing test.

The bitumen X70 specimens were fabricated to a cylindrical shape with a radius of $r_{0}$ (i.e., $4 \mathrm{~mm}$ ), and a height of $h$ (i.e., $2 \mathrm{~mm}$ ) in all tests as shown in Figure 1. In order to examine the 
repeatability of the experiments, three replicates were tested at each experimental condition and additional replicates were added when the deviations of target dynamic moduli and phase angles were greater than 10 percent.

Figure 3 shows linear amplitude sweep (LAS) results conducted at $20^{\circ} \mathrm{C}$ and $10 \mathrm{~Hz}$ with the start and end amplitudes equalling to $0.01 \%$ and $100 \%$, respectively. Theoretically, the dynamic modulus and phase angle of the undamaged bitumen should keep the same at the linear amplitude sweep test. But practically, there are some factors which make the measured dynamic modulus and phase angle of the bitumen vary slightly, such as: 1) the amplitude of controlled strain applied on the bitumen sample does not ideally equal to the target value, which is much more obvious when the strain level ranges from $0.01 \%$ to $0.1 \%$; 2 ) the sample for the linear amplitude sweep test is not perfectly homogeneous. It also could be seen that at $20^{\circ} \mathrm{C}$, if the amplitude of strain was approximately over $1 \%$, the dynamic modulus started to decrease and the phase angle began to decrease, respectively, which implied that fatigue damage of the bitumen was introduced. Therefore, in the LAS loading sequence, $0.1 \%$ and $0.6 \%$ (both less than $1 \%$ ) were selected as the start and end amplitudes to identify $\left|G^{*}\right|$ and $\delta_{0}$ of the undamaged bitumen $\mathrm{X} 70$ under the condition of $20^{\circ} \mathrm{C}$ and $10 \mathrm{~Hz}$.

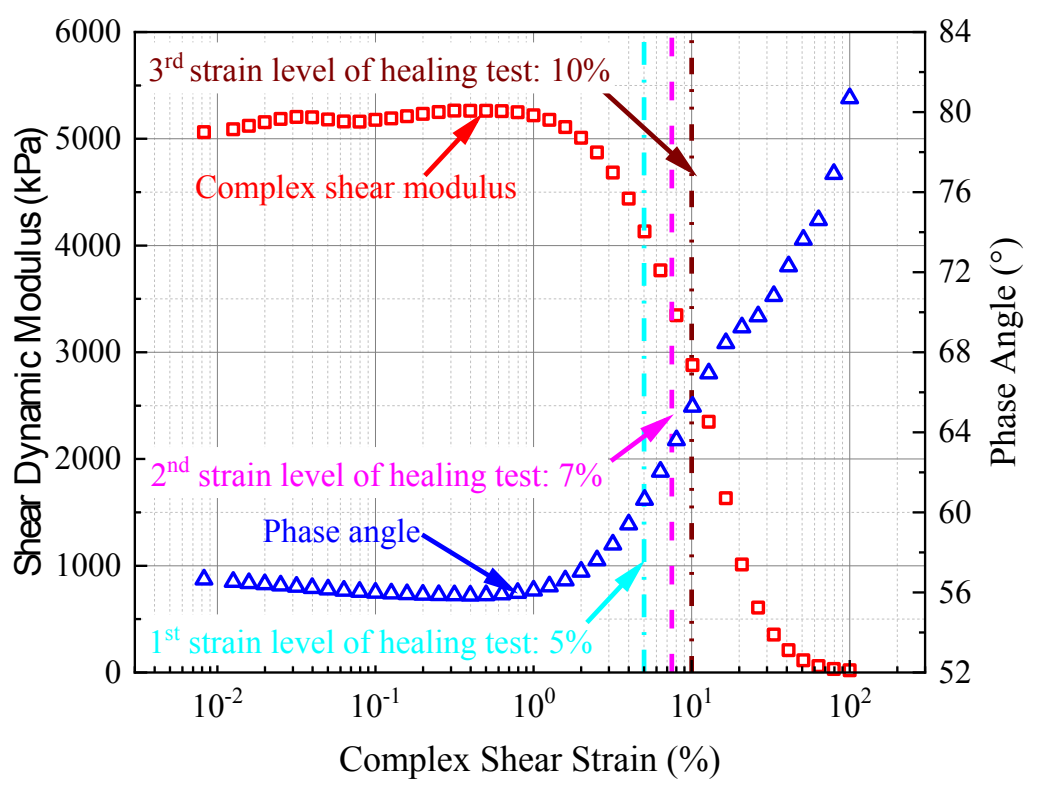

Fig. 3. Typical linear amplitude sweep (LAS) results at $20^{\circ} \mathrm{C}$ and $10 \mathrm{~Hz}$ and the selection of three strain levels for healing test.

To eliminate the sample-to-sample variation, the LAS test was followed by the time sweep healing test on the same bitumen sample. Figure 4 shows a schematic plot of loading sequences employed herein. The LAS test was conducted at strain levels from $0.1 \%$ and $0.6 \%$ to avoid any damage introduced to the sample. The LAS test results were used to determine the viscoelastic dynamic shear modulus and phase angle at the undamaged condition. Then the same sample was subjected to the time sweep healing test loading including a straincontrolled TS fatigue loading plus a rest period plus another strain-controlled TS loading. The amplitudes of strain and rest periods in the healing test were selected at different levels, explained as follows. 


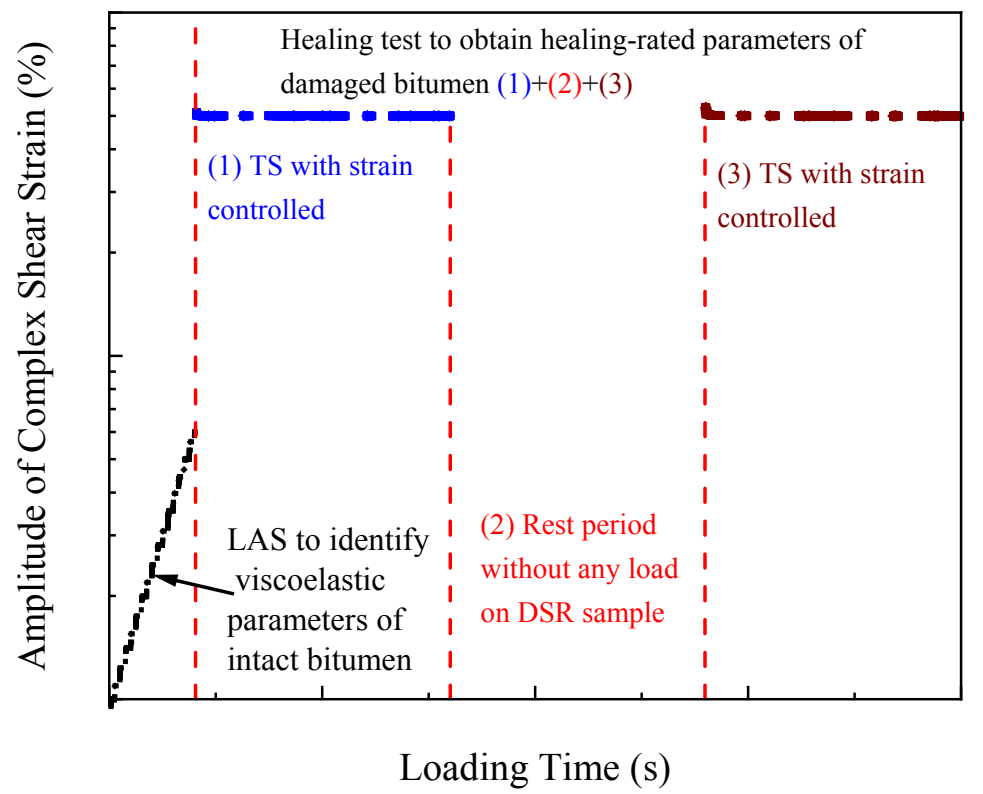

Fig. 4. Schematic illustration of input strain amplitude of LAS and healing tests

To evaluate healing properties of the bitumen X70 under different damage levels, three strain levels $(5 \%, 7 \%$ and 10\%) shown in Figure 3 were adopted to produce different crack lengths when the healing rests all started at $20 \mathrm{~min}$ (i.e., the first time sweep test lasted for $20 \mathrm{~min}$ ). $5 \%$ and $7 \%$ strain levels were previously utilised to predict the crack growth of bitumen sample in the time sweep test (Zhang and Gao, 2019), and the healing test designed in this paper was consist of two individual time sweep tests with a rest period separating them. Hence, strain levels of $5 \%$ and $7 \%$ were reselected to investigate the healing property of bitumen. The strain levels of $3 \%$ and $10 \%$ are other two options, which can be potentially utilised to evaluate the effect of damage level on healing properties. After conducting some trials of the strain level of 3\%, the author cannot obtain additional useful information that was different from the one of 5\% strain level. Accordingly, the strain level of $10 \%$ together with $5 \%$ and $7 \%$ was selected to investigate the impact of strain level on healing performance. Different damage levels were also introduced by applying the same strain level (5\%) but starting the healing rests at three different times ( $5 \mathrm{~min}, 10 \mathrm{~min}$ and $20 \mathrm{~min}$ ). To create the healing rate curve, different rest durations were used including $5 \mathrm{~s}, 10 \mathrm{~s}, 0.5 \mathrm{~min}, 1 \mathrm{~min}, 2 \mathrm{~min}$, $5 \mathrm{~min}, 10 \mathrm{~min}, 20 \mathrm{~min}$, and 40min. Therefore, the healing test plan in this paper can be summarized in Table 2.

Table 2 Healing Test Plan of the bitumen X70

\begin{tabular}{|l|l|l|l|l|}
\hline $\begin{array}{l}\text { Temperature } \\
\left({ }^{\circ} \mathbf{C}\right)\end{array}$ & Rest duration & $\begin{array}{l}\text { Amplitude of } \\
\text { Shear Strain (\%) }\end{array}$ & Healing happens @ & $\begin{array}{l}\text { Frequency } \\
(\mathbf{H z})\end{array}$ \\
\hline 20 & $\begin{array}{l}\text { 5s, 10s, 0.5 min, 1 min, 2min, } \\
5 \mathrm{~min}, 10 \mathrm{~min}, 20 \mathrm{~min}, 40 \mathrm{~min}\end{array}$ & $5,7,10$ & 5min, 10min, 20min & 10 \\
\hline
\end{tabular}

It is noted that, strain-controlled test instead of the stress-controlled one was selected due to that the crack grows in a much faster rate in the stress-controlled test, which makes the test results unstable or unmeasurable. In the strain-controlled test, the crack growth is more stable and test results can be well replicated. Hence, the authors select the strain-controlled mode of damage/healing test rather than the stress-controlled one.

\section{Results and Discussion}

4.1 Healing Index Characterised by Dissipated Pseudo Strain Energy 
Figure 5 shows the DPSE-based healing index calculated by Equation (8) at different damage levels and rest durations. In Figure 5, 5\%,7\%, and 10\% shown in the legend denote the amplitudes of the shear strains, and@5min,@10min, and@20min signify that the durations of the first TS test before the rest are 5min, 10min, and 20min, respectively. It is understood that higher amplitude of the shear strain or longer duration of the TS test will introduce higher cracking damage to the sample. It can be found in Figure 5 that when the duration of the first TS test (before healing) is 5 min (indicating lower cracking damage level), $\% H I_{D}$ were negative. This implies that there is no recovery of DPSE observed after the rest period, which means that the DPSE-based healing index could not characterise the healing behaviour at this relatively low cracking damage level. As the duration of the first TS test became longer, e.g., $10 \mathrm{~min}$ or $20 \mathrm{~min}$, indicating higher cracking damage, $\% H I_{D}$ gradually became positive. This implies that there is a clear recovery of DPSE after the rest period of healing test. One also finds that the standard deviation of $\% H I_{D}$ is as high as $60 \%$ at the low level of damage and when the damage in the bitumen sample enlarges, the standard deviation of $\% H I_{D}$ was reduced significantly (e.g., between $0.07 \%$ and $0.90 \%$ at the condition of 10\%@20min).

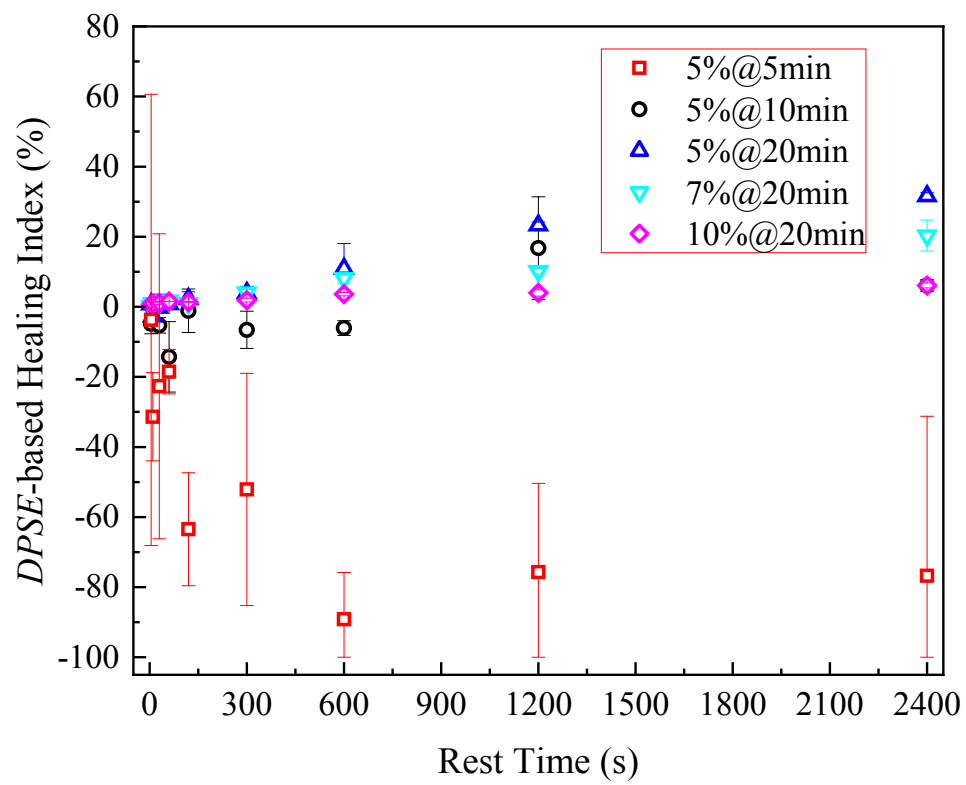

Fig. 5. DPSE-based healing index for bitumen at different testing conditions (5\%, 7\% and $10 \%$ are the strain levels of time sweep fatigue tests before rest period; @ $5 \mathrm{~min}, @ 10 \mathrm{~min}$ and @ $20 \mathrm{~min}$ are the time durations of the time sweep fatigue test before the rest period)

This phenomenon can be explained as follows: DPSE was determined by integrating the stress over the pseudo strain which is calculated by a linear viscoelastic constitutive equation. Thus, the DPSE includes all energy dissipation except that due to the linear viscoelastic relaxation. Hence, the DPSE has included the energy dissipation due to the viscoelastic nonlinearity, heat loss and cracking damage. Therefore, when the cracking damage is relatively low due to short loading times or low amplitudes of the shear strain (e.g. 5\%), the energy dissipation for crack initiation and propagation only consumes a small part of DPSE, most of which is used to generate nonlinear viscoelasticity and heat loss. In this case, using $D P S E$ to quantify the healing behaviour of the cracks would have become improper. In comparison, at high damage level (e.g., 10\%@20min), cracking damage dominates the mechanical behaviour of the bitumen sample and contributes most of the energy dissipation, then the DPSE-based healing index becomes more proper to quantify the healing behaviour although it is still not the real or direct parameter for healing characterisation duo to the inclusion of nonlinear viscoelasticity and heat loss in the DPSE. 
In sum, the DPSE may not be an accurate or reliable material parameter to characterise healing in bitumen as it inherently includes the energy dissipation for not only cracking growth but viscoelastic relaxation and heat loss. A material parameter quantifying the cracking performance only is preferred for healing characterisation.

As mentioned above, $\% H I_{D}$ is positive, and the standard deviation of $\% H I_{D}$ is comparatively small at high damage level (i.e., 7\%@20min, and 10\%@20min). Thus, all these data will be reserved to compare with $S$-based and $C L$-based healing indices, and to see if $\% H I_{D}$ at high damage levels can effectively capture the behaviour of healing in DSR-related healing tests.

\subsection{Healing Index Characterised by Pseudo Shear Stiffness and Crack Length}

Figure 6 shows the relationships between healing index and rest time when the fatigue healing test was conducted at $20^{\circ} \mathrm{C}$ and $10 \mathrm{~Hz}$. It is found that both $S$-based and $C L$-based healing indices grow as the increase of rest duration. In the short-term healing (rest time is short), the healing index develops quickly. As the healing time advances, the rate of healing index decreases, which is consistent with the existing knowledge shown in Equation (10). Comparing Figure 6 with Figure 5, one can find that the $S$ - or $C L$-based healing indices have much less variation than the DPSE-based healing index, for both low and high damage levels introduced by short and long TS fatigue loading time. This proves that the $S$ - or $C L$-based healing indices are more reliable and consistent material parameters to characterise the healing behaviour of the bitumen than the DPSE-based healing index.

It is also clearly demonstrated by Figure 6 that all $C L$-based healing indices are consistently greater than the $S$-based healing indices at all three different damage levels. As the cracking damages increase due to the extended duration of the first TS test in the healing test, difference between $\% H I_{D}$ and $\% H I_{C}$ is increased, much of which comes from long-term healing (rest time is longer). It is believed that the healing index is underestimated if pseudo stiffness is used to characterise the healing behaviour. Fundamentally, this is due to that the consistent drop of $S$ during the first TS fatigue test before the rest is ascribed to not only fatigue crack but nonlinear viscoelasticity and heat loss. In other words, the drop of $S$ was not solely caused by the cracking damage, leading to an increased denominator in the healing index model (Equation 6) and an underestimated healing index.
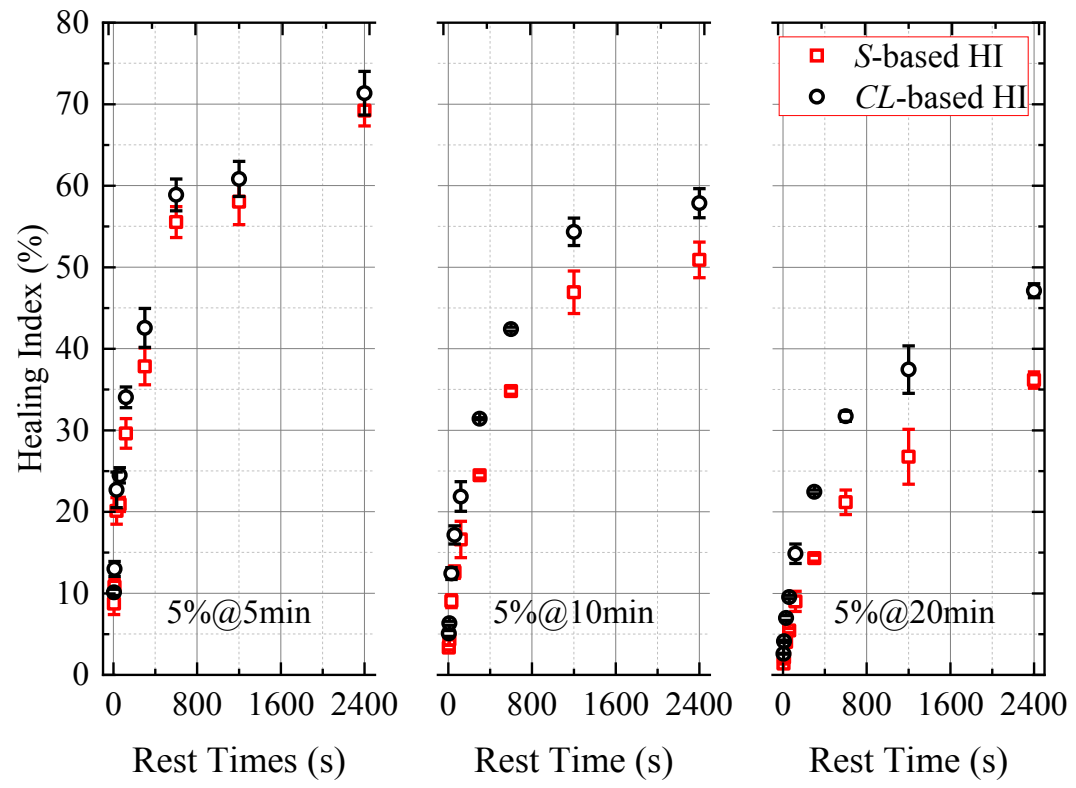

Fig. 6. Pseudo stiffness $(S)$ and crack length $(C L)$ based healing indices at different testing conditions (5\% is the strain level of time sweep fatigue tests before rest period; @ $5 \mathrm{~min}$, 
@10min and @20min are the time durations of the time sweep fatigue test before the rest period)

In summary, Neither the parameters of $S$ nor DPSE are the direct parameters for healing characterisation, and the inclusions of nonlinear viscoelasticity and heat loss will affect the applicability of $S$ and DPSE into healing characterisation. Healing can essentially be regarded as crack reduction, which microscopically originates from molecular chain approach, diffusion and randomisation. Hence, crack length can be regarded as a direct parameter to characterise healing performance. In addition, according to the law of energy conservation, the work done by external shear load in the DSR test equals to the stored and dissipated energies (include viscoelasticity, heat loss, crack initiation and propagation) of bitumen sample. Therefore, $C L$-based healing index can effectively exclude the effect of viscoelasticity and energy heat loss on the characterisation of healing performance.

\subsection{Healing Index at Different Levels of Cracking Damage}

The bitumen samples were damaged by applying different amplitudes of the shear strain $(5 \%$, $7 \%$, and $10 \%$, respectively) to investigate how the different levels of cracking damage affect the healing index of bitumen. Figure 7 shows the $S, C L$, and DPSE-based healing indices, for the bitumen X70 at the condition of $20^{\circ} \mathrm{C}, 10 \mathrm{~Hz}$ and @20min. It can be seen from Figure 7 that a higher amplitude of the shear strain yields a lower healing with all other conditions remaining the same in the designed healing test. The underpinning reason for this observation can be attributed to that a higher amplitude of the shear strain can introduce a higher cracking damage, which results in a less healing in the material. According to the DSR crack length model in Equation (5), the crack sizes introduced by the 20min TS fatigue test at $10 \mathrm{~Hz}$ have reached to $1.17 \mathrm{~mm}, 1.52 \mathrm{~mm}$, and $2.30 \mathrm{~mm}$ before the rest period when the strain amplitudes were $5 \%, 7 \%$, and $10 \%$, respectively. The higher cracking damage will lead to a quicker degradation of the pseudo shear stiffness, more consumption of the dissipated pseudo strain energy, and bigger and more interconnected cracks. According to the theory of healing proposed by Wool and O'Conner (1981), the cracked interfaces can be rearranged, approached, wetted, diffused and randomized during the rest period, however, these processes would be delayed, slowed down or even prevented due to a greater distance between the material molecules on the two cracked surfaces when the crack size is big enough. Therefore, the bigger cracks reduce the healing rate and even prevent healing happening. Therefore, the healing index always declines as the increase of cracking damage levels (e.g., due to the increase of the amplitude of the shear strain). 

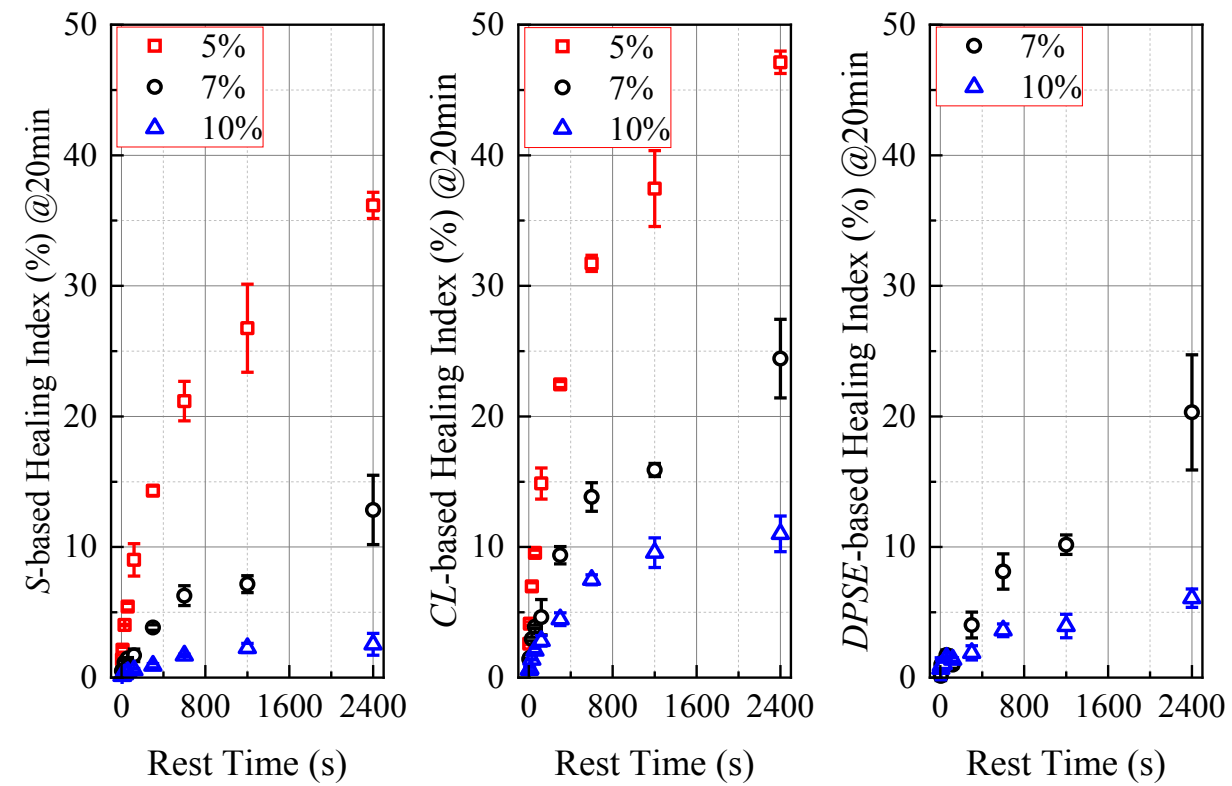

Fig. 7. Healing index of the bitumen X70 at 20 $\left.{ }^{\circ} \mathrm{C}\right) / 10 \mathrm{~Hz} / @ 20 \mathrm{~min}$ and different amplitudes of the shear strain

Figure 7 also shows that $\% H I_{C}$ is always greater than the $\% H I_{S}$ and $\% H I_{D}$ at each of the applied strain amplitudes. The reason remains the same with the one explained in sections 4.1 and 4.2, namely, the change of $S$ or DPSE in the first TS fatigue loading period was caused by not only fatigue cracking but nonlinear viscoelasticity and heat loss, making a larger denominator of the healing index model in Equation (6) and therefore a lower healing indices. In comparison, using cracking length to characterise the fatigue damage and healing has distinguished the healing from all other physics such as viscoelasticity and heat loss, which can provide a direct and accurate characterisation of the healing for the material. Additionally, it is noteworthy that the amplitude of the shear strain influences both short-term and long-term healing rates impressively, details of which will be analysed in the following section.

\subsection{Healing Rate Modelling by Ramberg-Osgood Model}

Once the $S$ - and $C L$-based healing indies determined, the parameters of $\dot{h}_{1}, \dot{h}_{2}$, and $h_{\beta}$ in Equations (10) can be fitted by nonlinear regression method, and the results are graphically represented in Figure 8. Note that the DPSE-based healing index is not used to model the healing rate in this section, since the DPSE is not recommended as a reliable parameter to characterise the healing of the bitumen (especially in low damage level), suggested by the analysis in Section 4.1. 


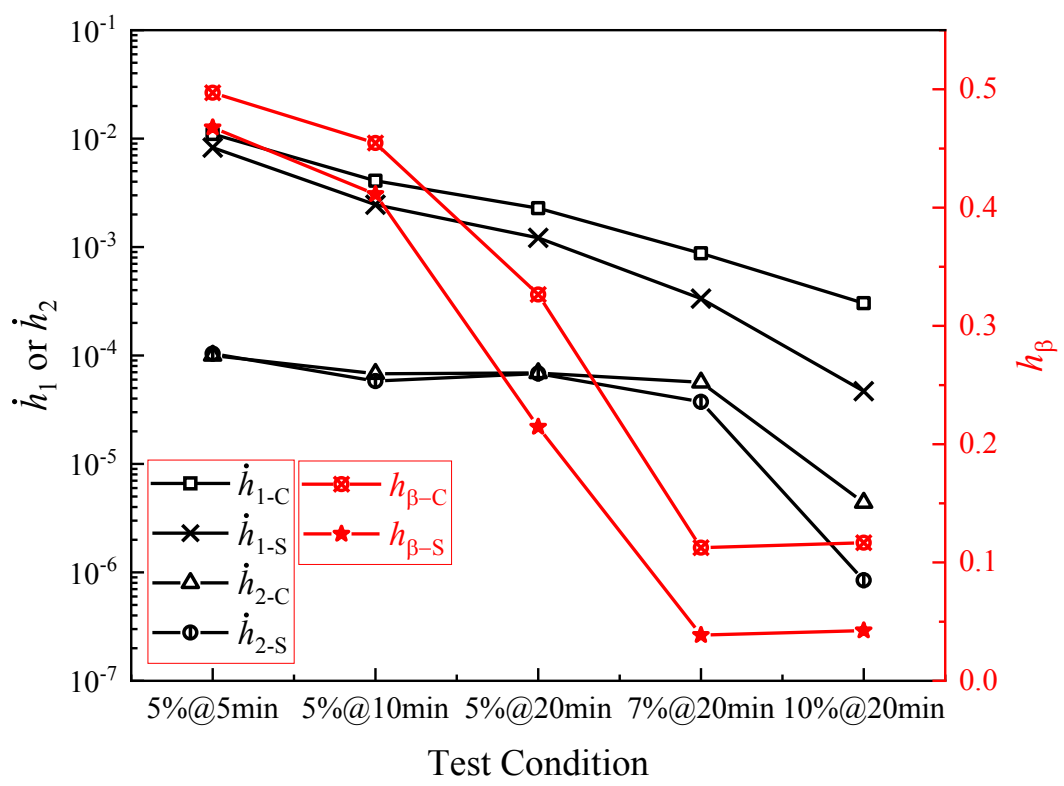

Fig. $8 . \dot{h}_{1}, \dot{h}_{2}$, and $h_{\beta}$ characterised by $C L$ and $S\left(\dot{h}_{1-C}\right.$ and $\dot{h}_{1-S}$ are $C L$ - and $S$-based shortterm healing rates; $\dot{h}_{2-C}$ and $\dot{h}_{2-S}$ are $C L$ - and $S$-based long-term healing rates; and $h_{\beta-C}$ and $h_{\beta-S}$ are $C L$ - and $S$-based short-term healing potentials.)

Figure 8 shows that, $\dot{h}_{1-C}\left(C L\right.$-based short-term healing rate) is consistently higher than $\dot{h}_{1-S}$ ( $S$-based short-term healing rate), which implies that the healing will be underestimated when characterised by the pseudo stiffness. Again, this is due to that the change of pseudo stiffness during the fatigue period resulted from not only fatigue cracking, but the nonlinear viscoelasticity and the heat loss. Recovery of the three physics would be slower and taking longer time, which leads to a smaller healing rate. The same observations are identified for the long-term healing rate and the healing potential, namely, the $\dot{h}_{2-C}$ is always greater than $\dot{h}_{2-S}$ and the $h_{\beta-C}$ is always higher than $h_{\beta-S}$ at all test conditions. This indicates that the exclusion of the non-cracking related physics such as nonlinear viscoelasticity and heat loss is fundamentally needed to accurately evaluate healing potential of bitumen. It is desired to accurately characterise the healing behaviour using the cracking-related parameter such as the cracking length which has excluded the effect of those physics other than cracking.

Note that the damage in the DSR test is quantified by the cracking length, which have achieved $0.52 \mathrm{~mm}, 0.84 \mathrm{~mm}, 1.17 \mathrm{~mm}, 1.52 \mathrm{~mm}$, and $2.30 \mathrm{~mm}$ at the end of the first TS fatigue loading period before the rest at the test conditions of 5\%@5min, 5\%@10min, 5\%@20min, 7\%@20min and 10\%@20min, respectively. One can see that the longer fatigue loading before the rest or the higher strain levels in the fatigue test, the longer cracking length indicating a higher cracking damage. Figure 8 shows that the healing parameters (i.e., $\dot{h}_{1}, \dot{h}_{2}$, and $h_{\beta}$ ) decrease as the damage increases, which suggests that the a severe cracking damage will have a significantly small healing rate. It is also noted that, for the healing potential, both $h_{\beta-C}$ and $h_{\beta-S}$ at the condition of 7\%@20min changed very little from the ones at the condition of 10\%@20min, which is due to the fact that at the condition of 7\%@20min, bitumen sample is damaged so severely that further damage (i.e., 10\%@20min) cannot effectively reduce the achievable healing. This phenomenon can be explained that as the crack propagates, distance between two cracked surfaces increases (shown in Figure 1) under the continuous rotational shear loads, which in turn dramatically reduce the electrostatic force 
(inversely proportional to the effective distance between polar molecules from the two cracked surfaces) and van der Waals force (inversely related to the effective distance between non-polar molecules from the two cracked surfaces) (Gao et al., 2019). Therefore, a higher cracking damage will lead to a lower healing rate for both short-term and long-term healing rates. When the damage of the sample approaches to a critical state where the distance between the two cracked surface has been critically high enough, the healing driving forces including the electrostatic and Vander Waals forces become so weak that the two cracked surface in the bitumen cannot be rearranged, wetted or diffused, leading to the unhealable cracks at such a highly damaged condition. Therefore, the healing potential would not change significantly in a severely damaged material.

After the obtainment of $\dot{h}_{1}, \dot{h}_{2}$, and $h_{\beta}$ at different healing tests, healing rate can be predicted by Equation (10) and shown in Figure 9. It can be found that the healing rate is relatively higher when rest time is short, but it drops quickly and eventually approaches to $\dot{h}_{2}$. In addition, as explained before, healing rate declines gradually as the development of damage level.

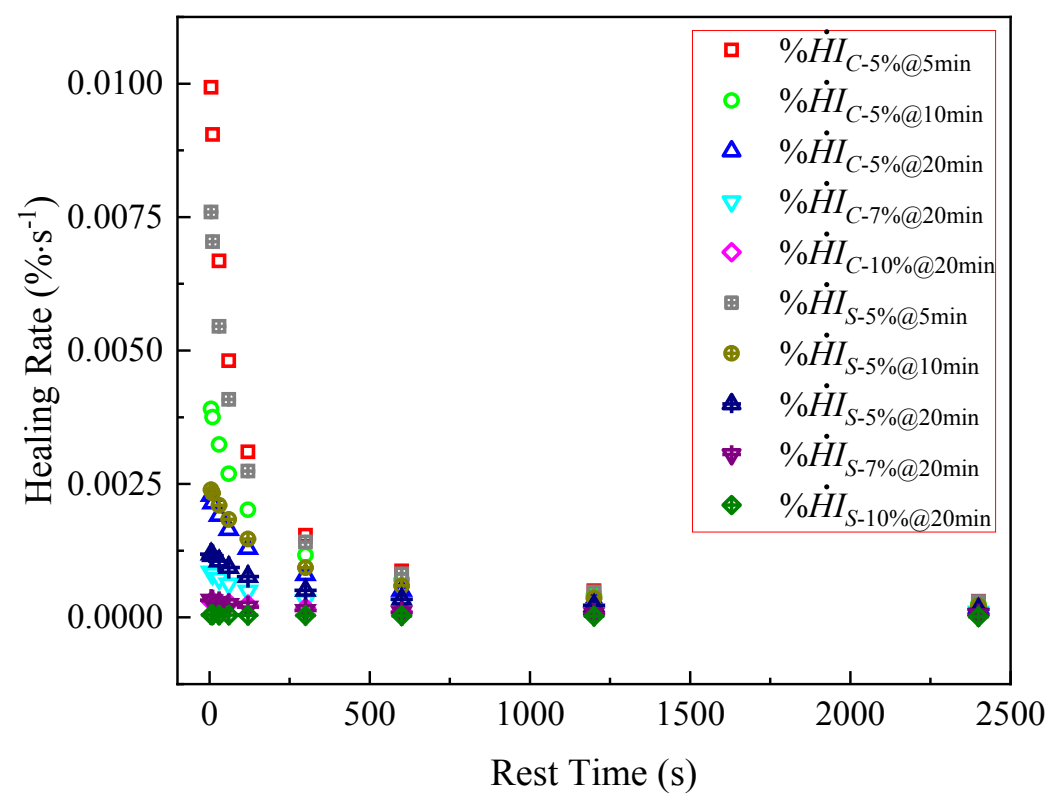

Fig. 9. Trends of healing rate in the process of rest period

\section{Summary and Conclusions}

This paper investigated the impact of the cracking damage levels on the healing properties of a bitumen binder. The healing was characterised by three different material parameters including pseudo shear stiffness $(S)$, dissipated pseudo strain energy (DPSE), and crack length $(C L)$ in the healing test designed on the base of DSR test. Firstly, a new formation of normalized healing index was proposed using the $S, D P S E$, and $C L$ values before and after the rest period. Then, healing of the bitumen was characterised under different rest periods $(5 \mathrm{~s}, 10 \mathrm{~s}, 0.5 \mathrm{~min}, 1 \mathrm{~min}, 2 \mathrm{~min}, 5 \mathrm{~min}, 10 \mathrm{~min}, 20 \mathrm{~min}$, and $40 \mathrm{~min}$ ), different amplitudes of the shear strain $(5 \%, 7 \%$, and $10 \%)$, and different loading times (5min, $10 \mathrm{~min}$ and $20 \mathrm{~min})$ for the time sweep fatigue loading before the rest at $20^{\circ} \mathrm{C}$ and $10 \mathrm{~Hz}$. Finally, the applicability and accuracy of $S$-, DPSE-, and $C L$-based healing index were presented, and the superiority of $C L$-based healing properties was also analysed. The main findings and conclusions of this paper are summarized as follows:

(1) The negative and high standard deviation of the DPSE-based healing index indicates the inapplicability of the DPSE to characterise the healing behaviour of the bitumen, particularly 
when the damage level is relatively low.

(2) $C L$-based healing index is fundamental and more reliable to characterise the healing properties of the bitumen. $S$-based and DPSE-based (at high damage level) healing indices underestimate the healing performance of the bitumen, which make the short-term and longterm healing rates, and the healing potential smaller than their real values.

(3) Healing index increases as the advance of rest duration and can be essentially modelled by Ramberg-Osgood equation. Longer fatigue test before rest duration and higher amplitude of shear load will yield more damage of bitumen sample in the healing test. Healing index drops as the increase of the cracking damage level, which leads to the decreased short-term and long-term healing rates, and the reduced healing potential as well. When the bitumen sample is severely damaged, further addition of the damage level cannot decrease the healing potential anymore.

\section{Conflict of interest}

The authors declared that there is no conflict of interest.

\section{Acknowledgements}

The authors would like to acknowledge the financial support from Marie Skłodowska-Curie Individual Fellowships under EU's H2020 programme (Grant No. 789551), the National Nature Science Foundation of China (Grant No. 51978229), Fundamental Research Funds for the Central Universities in China (Grant Nos. JZ2018HGTB0258), AIMR Seedcorn Grant from Aston University (Grant No. 201901), the National Nature Science Foundation of China (Grant No. 51408173), and China Postdoctoral Science Foundation funded Project (Grant No. 2015M571928).

\section{References}

Ayar, P., Moreno-Navarro, F., Rubio-Gámez, M.C., 2016. The healing capability of asphalt pavements: a state of the art review. Journal of Cleaner Production 113, 28-40.

Bazin, P., Saunier, J., 1967. Deformability, fatigue and healing properties of asphalt mixes, In: Proceedings of the 2nd International Conference on the Structural Design of Asphalt Pavements. Ann Arbor, Michigan, USA.

Canestrari, F., Virgili, A., Graziani, A., Stimilli, A., 2015. Modeling and assessment of selfhealing and thixotropy properties for modified binders. International Journal of Fatigue 70, 351-360.

Carpenter, S.H., Shen, S., 2006. Dissipated energy approach to study hot-mix asphalt healing in fatigue. Transportation Research Record 1970(1), 178-185.

Cheng, D., Little, D.N., Lytton, R.L., Holste, J.C., 2002. Surface energy measurement of asphalt and its application to predicting fatigue and healing in asphalt mixtures. Transportation Research Record 1810(1), 44-53.

Ganjei, M.A., Aflaki, E., 2019. Application of nano-silica and styrene-butadiene-styrene to improve asphalt mixture self healing. International Journal of Pavement Engineering 20(1), 89-99.

Gao, Y., Zhang, Y., Yang, Y., Zhang, J., Gu, F., 2019. Molecular dynamics investigation of interfacial adhesion between oxidised bitumen and mineral surfaces. Applied Surface Science 479, 449-462.

Garcia, A., 2012. Self-healing of open cracks in asphalt mastic. Fuel 93(1), 264-272.

Hintz, C., Bahia, H., 2013. Understanding mechanisms leading to asphalt binder fatigue in the dynamic shear rheometer. Road Materials and Pavement Design 14(sup2), 231251. 
Kim, Y.R., Little, D.N., Benson, F.C., 1990. Chemical and mechanical evaluation on healing mechanism of asphalt concrete (with discussion). Journal of the Association of Asphalt Paving Technologists 59, 240-275.

Koohi, Y., Luo, R., Lytton, R.L., Scullion, T., 2013. New Methodology to Find the Healing and Fracture Properties of Asphalt Mixes Using Overlay Tester. Journal of Materials in Civil Engineering 25(10), 1386-1393.

Kringos, N., Scarpas, A., Pauli, T., Robertson, R., 2009. A thermodynamic approach to healing in bitumen. Taylor and Francis Group London.

Lee, J.S., Lee, S., Kim, Y.R., 2011. Evaluation of healing effect by rest periods on asphalt concrete slab using MMLS3 and NDE techniques. KSCE Journal of Civil Engineering 15(3), 553-560.

Li, C., Wu, S., Chen, Z., Tao, G., Xiao, Y., 2018. Enhanced heat release and self-healing properties of steel slag filler based asphalt materials under microwave irradiation. Construction and Building Materials 193, 32-41.

Little, D.N., Lytton, R.L., Williams, D., Kim, Y.R., Assoc Asphalt Paving Technol; Assoc Asphalt Paving, T., 1999. An analysis of the mechanism of microdamage healing based on the application of micromechanics first principles of fracture and healing.

Liu, Q., Tang, J., He, L., 2016. Thermally Activated Healing of Fatigue Damage in Asphalt Binders. Journal of Testing and Evaluation 45(2), 574-581.

Liu, Q.T., Tang, J., He, L., 2017. Thermally Activated Healing of Fatigue Damage in Asphalt Binders. Journal of Testing and Evaluation 45(2), 574-581.

Luo, X., Birgisson, B., Lytton, R.L., 2019. Kinetics of healing of asphalt mixtures. Journal of Cleaner Production, 119790.

Luo, X., Lytton, R.L., 2015. Characterization of healing of asphalt mixtures using creep and step-loading recovery test. Journal of Testing and Evaluation 44(6), 2199-2210.

Lytton, R., Chen, C., Little, D., 2001. Microdamage healing in asphalt and asphalt concrete, volume III: A micromechanics fracture and healing model for asphalt concrete.

Lytton, R.L., 2000. Characterizing asphalt pavements for performance. Transportation Research Record 1723(1), 5-16.

Mannan, U.A., Ahmad, M., Tarefder, R.A., 2017. Influence of moisture conditioning on healing of asphalt binders. Construction and Building Materials 146, 360-369.

Norambuena-Contreras, J., Yalcin, E., Garcia, A., Al-Mansoori, T., Yilmaz, M., HudsonGriffiths, R., 2018. Effect of mixing and ageing on the mechanical and self-healing properties of asphalt mixtures containing polymeric capsules. Construction and Building Materials 175, 254-266.

Palvadi, S., Bhasin, A., Little, D.N., 2012. Method to quantify healing in asphalt composites by continuum damage approach. Transportation Research Record 2296(1), 86-96.

Patti, F., Mansour, K., Pannirselvam, M., Giustozzi, F., 2018. Mining materials to generate magnetically-triggered induction healing of bitumen on smart road pavements. Construction and Building Materials 171, 577-587.

Qiu, J., Van de Ven, M., Wu, S., Yu, J., Molenaar, A., 2011. Investigating self healing behaviour of pure bitumen using dynamic shear rheometer. Fuel 90(8), 2710-2720.

Ramberg, W., Osgood, W.R., 1943. Description of stress-strain curves by three parameters.

Si, Z., Little, D., Lytton, R., 2002. Characterization of microdamage and healing of asphalt concrete mixtures. Journal of materials in civil engineering 14(6), 461-470.

Si, Z.S., Little, D.N., Lytton, R.L., 2002. Evaluation of fatigue healing effect of asphalt concrete by pseudostiffness. Transportation research record 1789(1), 73-79.

Sun, D., Yu, F., Li, L., Lin, T., Zhu, X., 2017. Effect of chemical composition and structure of asphalt binders on self-healing. Construction and Building Materials 133, 495-501. 
Tabakovic, A., Schlangen, E., 2016. Self-Healing Technology for Asphalt Pavements, in: Hager, M.D., VanDerZwaag, S., Schubert, U.S. (Eds.), Self-Healing Materials. pp. 285-306.

Tan, Y., Shan, L., Kim, Y.R., Underwood, B.S., 2012. Healing characteristics of asphalt binder. Construction and Building Materials 27(1), 570-577.

Wool, R., O'connor, K., 1981. A theory crack healing in polymers. Journal of Applied Physics 52(10), 5953-5963.

Zhang, Y., Gao, Y., 2019. Predicting crack growth in viscoelastic bitumen under a rotational shear fatigue load. Road Materials and Pavement Design, 1-20. 